\title{
Chronic Liver Failure after Treatment with Infliximab for Ankylosing Spondylitis in a Patient with Hepatitis B
}

\author{
Yun-ru Li, Feng-xin Chen, Xue-fei Duan, Xue-song Gao and Xiao-ling Fan
}

A 50-year-old man with ankylosing spondylitis was treated successfully with infliximab, who was also a HBV carrier for about twenty five years. After injection with infliximab for four times, he developed jaundice and HBV DNA was detectable in serum. Serum aminotransferase and total bilirubin levels were higher than normal. Then he was hospitalized and treated with entacavir and Chinese herb medicine. But his liver damage aggravated and was diagnosed as acute on chronic liver failure. Finally, liver transplantation was carried out and he was cured successfully.

Key words: Acute on chronic liver failure; Hepatitis B; Ankylosing spondylitis

$\mathrm{A}$ pproximately $7.18 \%$ people in the general population are HBsAg positive in China. The total number is about 100 million. $^{1}$ Part of these population suffered from autoimmune disease, kidney disease, carcinomas and organ transplantation. Some immunosuppressant such as prednisone were applied in treatment of these diseases. And some articles reported that hepatitis B virus (HBV) reactivation and liver failure occured during treatment with immunosuppressant. Tumor necrosis factor (TNF)alpha inhibitors is an important immunosuppressant, which has provided clinicians effective tool over autoimmune diseases. Along with the widespread usage of this medication, the side effect such as reactivation of latent infections was found. One of such infection is HBV infection. Despite of administration of nucleos $(t)-$ ide analogues to these patients, the prognosis was still disappointed.

\section{CASE REPORT}

A 50-year-old man suffered from ankylosing spondylitis was a HBV carrier for about twenty five years. He was treated with infliximab for ankylosing spondylitis. Before therapy, the patient never had liver injury and his serum HBV DNA was not detectable. After four months, laboratory findings showed liver injury and

\footnotetext{
Beijing Ditan Hospital, Capital Medical University, Beijing 100015, China

Correspondence: Yun-ru Li, E-mail: yunru2008@126.com Telephone: $+86-10-84322327$
}

reactivation of HBV. Finally, he was diagnosed as liver failure and received liver transplantation.

He was admitted to our hospital (March 5, 2010) due to "HBsAg positive for 25 years and weakness for 2 weeks". Two weeks before admission, fatigue, abdominal distention and weakness were found, then accompanied with nausea and vomiting. Liver function indexes were as following: ALT $652 \mathrm{U} / \mathrm{L}$, AST 1280 U/L, TBil $224 \mu \mathrm{mol} / \mathrm{L}$, DBil $126 \mu \mathrm{mol} / \mathrm{L}$. Physical examination: severe yellow sclera and skin, spider angiomas and liver palm, untouched liver, enlarged spleen under ribs, pain in liver area. And shifting dullness was negative.

Approximately sixteen years before admission, the patient was diagnosed as "ankylosing spondylitis" with back pain in Beijing Union Medical College Hospital. At first, he was treated with Celecoxib (200 mg, bid ) and leflunomide (20 mg, qd) alternately. Four months before admission, his back pain aggravated and he was treated with infliximab $(4 \mathrm{mg} / \mathrm{kg}$ ). His back pain relieved after the first injection and disappeared after the second injection at the second week. Thereafter, the third and fourth injections were administered at the 6 th and the 12 th week, respectively. Before treatment with infliximab, his liver function was normal and HBV DNA was undetectable without antiviral therapy. After the four injections, his liver function was exacerbated. In addition, the patient's brother also suffered from "ankylosing spondylitis".

The laboratory results showed: ALT $421 \mathrm{U} / \mathrm{L}$, AST 
Dynamic change of PTA and INR

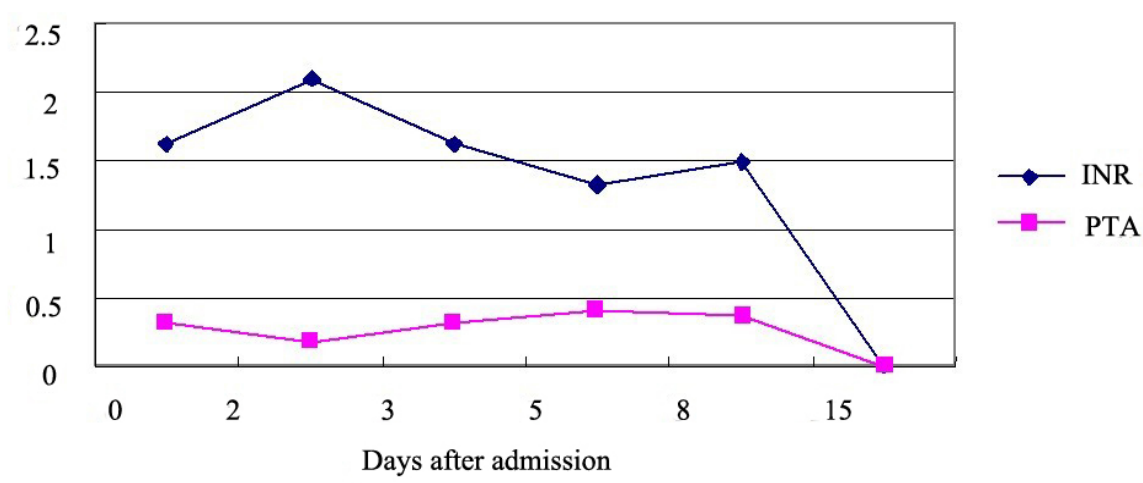

A

Dynamic change of liver function idex

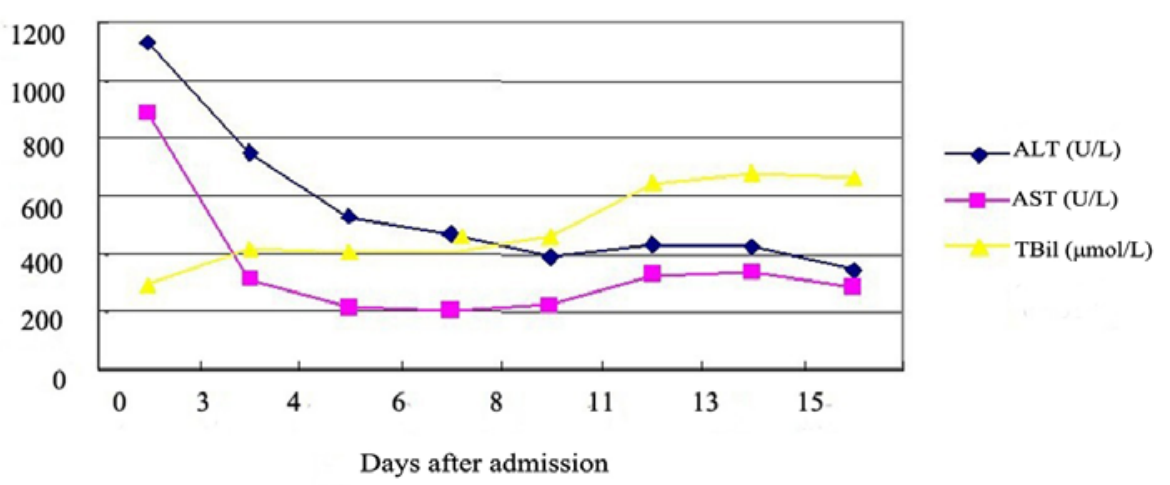

B

Dynamic change of HBV DNA level

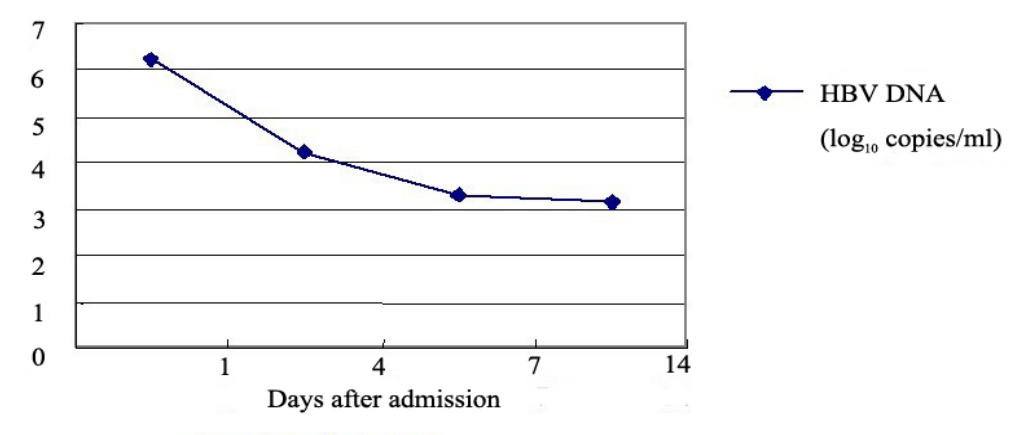

C

Figure 1. Dynamic changes of PTA and INR, indexes of liver function and HBV DNA.

Notes: A: PTA and INR decreased until undetectable before liver transplantation; B: ALT and AST level decreased and TBil level increased with liver inflammation aggravated. C: HBV DNA level decreased after treatment with entecavir.

$335 \mathrm{U} / \mathrm{L}$, TBil $676.1 \mu \mathrm{mol} / \mathrm{L}$, DBil $428.8 \mu \mathrm{mol} / \mathrm{L}$, TP $66.7 \mathrm{~g} / \mathrm{L}$, ALB $34.3 \mathrm{~g} / \mathrm{L}$, GLB $26.8 \mathrm{~g} / \mathrm{L}$, AFP $48.2 \mathrm{ng} /$ L, PTA $19.8 \%$, HBV DNA $2.49 \times 10^{6}$ copies $/ \mathrm{ml}$, HBsAg $>250 \mathrm{IU} / \mathrm{L}$, anti-HBs $0 \mathrm{IU} / \mathrm{L}, \mathrm{HBeAg} 0.28 \mathrm{~S} / \mathrm{CO}$, antiHBe $0.01 \mathrm{~s} / \mathrm{co}$, anti-HBc $14.70 \mathrm{~s} / \mathrm{co}$. CT manifestations: the shape and size of liver was normal, with smooth envelope, and the diameter of portal vein was $1.2 \mathrm{~cm}$, spleen thickness was $3.2 \mathrm{~cm}$.

Clinical manifestation showed as following: (1) His weakness aggravated, eventually he had to sleep in bed. (2) Nausea and vomiting occured repeatedly. (3) Hypoglycemia occured every night. (4) Jaundice 
in his skin and sclera deepened. As a result, he was diagnosed as chronic liver failure. After admission, he received injections of compound glycirrhizin, reduced glutathione, polyene phosphatidylcholine, hepatocyte promoting growth factor and butanedisul fonate to protect liver function. And thymosin alpha was administered to enhance his immunology and entecavir was applied to inhibit virus replication. Although HBV DNA was undetectable, his liver necrosis aggravated progressively. Three weeks after admission, he received liver transplantation and still survived at present.

\section{DISCUSSION}

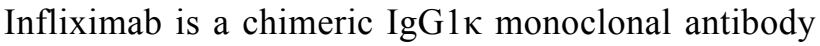
which is specific for human tumor necrosis factoralpha (TNF- $\alpha$ ). Its molecular weight is approximately $149.1 \mathrm{kD}$. It is produced by a recombinant cell line cultured by continuous perfusion and is purified by a series of steps including measures to inactivate and remove viruses. At present, infliximab is applied to treat rheumatoid arthritis, psoriatic arthritis, ulcerative colitis, Crohn's disease, and ankylosing spondylitis, especially when other medicines were not effective. Severe liver injury including acute liver failure and autoimmune hepatitis, has been rarely reported in patients without hepatitis $\mathrm{B}$ or $\mathrm{C}$ when receiving infliximab treatment. In clinical trials of rheumatoid arthritis, Crohn's disease, ulcerative colitis, ankylosing spondylitis, plaque psoriasis, and psoriatic arthritis, elevations of aminotransferases were observed (ALT more common than AST) in a greater proportion of patients receiving infliximab than in controls, but most patients were asymptomatic. ${ }^{2}$

Some reports showed that reactivation of hepatitis $B$ virus and liver damage occurred in patients receiving TNF-blocking agents, who were chronic HBV carriers. Total of 257 patients with HBV markers positive (89 $\mathrm{HBsAg}$ positive carriers, and 168 anti-HBc positive ones) who received anti-TNF therapy were analyzed. The results showed that $\mathrm{HBV}$ reactivation was reported in $35(39 \%) \mathrm{HBsAg}(+)$ carriers. And acute liver failure was reported in 5 patients, 4 of whom died. It was considered that infliximab was associated with a higher rate of liver damage ${ }^{3}$. Another famous report was on inflammatory bowel disease patients with HBsAg positive. Liver dysfunction was observed in 23 of the 134 (17.2\%) HBsAg positive patients. Prolonged immunosuppression (> 3 months) was an independent predictor of liver dysfunction [odds ratio (OR) 3.06; 95\% confidence interval (CI) $1.02-9.16] .{ }^{4}$
HBV reactivation is associated with a wide range of clinical manifestations after treatment with immunosuppressant, from asymptomatic to acute hepatitis that can be serious and cause liver failure. Most HBV reactivation has three phases: (1) replication, in which serum HBV DNA levels increased; (2) hepatitis, which happened 2-3 weeks after HBV DNA levels increased and was characterized by an elevation of transaminases, and sometimes, symptoms such as fatigue, malaise and jaundice. In some cases, hepatitis can be fulminant (hepatic encephalopathy, coagulopathy, etc.); (3) recovery, if the patient survived from fulminant hepatitis or had liver transplantation, then the infection moves to the third phase. $^{5}$

How to treat and prevent the side effects of HBV reactivation? Most reports showed lamivudine was the most commonly applied nucleoside analogue in treatment of HBV reactivation. It is capable of inhibiting HBV replication. However, at least one third of patients died of HBV reactivation despite of treatment with lamivudine. This might be due to its lower antiviral potency than other nucleos(t)ide analogues such as telbivudine, entecavir and tenofovir dipivoxil, but till now there are few data to prove the latter ones can also decrease mortality. These facts led to the conclusion that for such patients it would be better to administer prophylactic medication before treatment with immunosuppressant to prevent HBV reactivation, as it would hinder the development of hepatitis. ${ }^{6}$ But there are few reports about prophylactic medication. From 2003 to 2009, 17 articels reported that 21 patients experienced reactivation of hepatitis B when treated with infliximab, including 15 cases with inactive carrier state, 4 cases with chronic active hepatitis, 1 case with occult HBV infection and 1 case with delta virus co-infection. As a result, 6 cases who received lamivudine prophylacticly had no HBV reactivation, while other 15 patients had $\mathrm{HBV}$ reactivation, and 3 cases of them occured fatal fulminant hepatitis. But lamivudine prophylaxis is a long-term treatment and resistance to lamivudine may appear, particularly in patients with high HBV replication. Thus, it is preferable to use drugs with lower rates of resistance such as tenofovir dipivoxil or entecavir in these cases. ${ }^{5}$

\section{REFERENCES}

1. $\mathrm{Yu}$ W, Jia JD. Control of hepatitis B in China: prevention and treatment. Expert Rev Anti Infect Ther 2011; 9:21-25. 
2. Hanauer SB. Safety of infliximab in clinical trials. Aliment Pharmacol Ther 1999;13(suppl 4):16-22.

3. Pérez-Alvarez R, Díaz-Lagares C, García-Hernández F, LopezRoses L, Brito-Zerón P, Pérez-de-Lis M, et al. Hepatitis B virus (HBV) reactivation in patients receiving tumor necrosis factor (TNF)-targeted therapy: analysis of 257 cases. Medicine (Baltimore) 2011; 90:359-371.

4. Park SH, Yang SK, Lim YS, Shim JH, Yang DH, Jung KW, et al. Clinical courses of chronic hepatitis B virus infection and inflammatory bowel disease in patients with both diseases. Inflamm Bowel Dis 2012;18:2004-2010.

5. Manzano-Alonso ML, Castellano-Tortajada G. Reactivation of hepatitis B virus infection after cytotoxic chemotherapy or immunosuppressive therapy. World J Gastroenterol 2011; 17:1531-1537.

6. Hoofnagle JH. Reactivation of hepatitis B. Hepatology 2009;49(5 Suppl):S156-165. 